\title{
Preface to the special issue on energy utilization and conservation
}

\author{
LIAO Sheng-ming(廖胜明 $)^{1}$, SUN Zhi-qiang(孙志强 $)^{1}$, ZHOU Chenn(周谦 $)^{2}$
}

1. School of Energy Science and Engineering, Central South University, Changsha 410083, China; 2. Center for Innovation through Visualization and Simulation, Purdue University Northwest, Hammond, IN 46323, USA

(C) Central South University Press and Springer-Verlag GmbH Germany, part of Springer Nature 2019

Energy utilization and conservation are seen as important solutions to energy crisis and environmental pollution. New technologies, such as renewable energy utilization, high-efficiency conversion, energy management, and energy saving, are playing important roles in industry. However, improving performance and reducing costs in their implementation are still big challenges and therefore going to be hot topics. In recent years, significant research progresses on these topics have been made from fundamental studies to applicationoriented prototypes around the world. We organize this special issue "energy utilization and conservation" in Journal of Central South University to show the latest advances.

This special issue includes twenty-three original contributions. The topics cover theoretical study and application of multiphase flow. Flow characteristics at low Reynolds number around two in-line circular cylinders with slits was investigated by SUN Zhi-qiang and his group; SONG Yan-po et al reconstructed bubble profiles from gas-liquid two-phase flow data. Moreover, a propagation based thermal conductivity measurement method was developed by ZHOU Tian et al. We also thank to the authors' contributions in entropy analysis of flow statement in porous medium, and natural convection horizontal annular finned tube.

Six articles about energy system engineering and renewable energy are also included in this special issue. LIAO Sheng-ming and his group built an energy prediction model for urban energy consumption. MA Wei-wu et al evaluated and compared working fluids for organic Rankine cycles (ORCs) by developing group-contribution methods and second-law-based models. Also, LIU Li et al numerically studied the geothermal heat extracted by fluid flowing in buried tube and YIN et al experimentally studied the repair characteristics of damaged rock salt of underground gas storage.

Two articles focusing on combustion are also included. FAN Ai-wu and his group examined the effects of wall thickness and materials on flame stability in a planar micro-combustor. As well, catalytic combustion characteristic of hydrogen/air in the micro turbine engine was analyzed by $\mathrm{E}$ Jia-qiang et al.

In addition, several articles on heat and mass transfer in smelting, electrolysis and welding are also presented.

In summary, these contributions present the cutting-edge researches in the field of energy utilization and conservation. We would like to sincerely thank all the authors for their great efforts in preparing high-quality manuscripts. We also thank the professional editorial office of Journal of Central South University for their hard-works to make this issue such a great success.

\section{Guest editors}

\section{LIAO Sheng-ming}

Prof. LIAO obtained his B.S. and M.S. from Tsinghua University in 1985 and 1989, respectively, and $\mathrm{PhD}$ from Hong Kong University of Science and Technology in 2002. Since 2005, he has been a Professor in the School of Energy Science and Engineering, Central South University (CSU), China. His current research interests are focused on 
energy system engineering, new energy, energy saving technologies, and basic problems of thermal physics and energy utilization in engineering. As one of the earliest scholars to study trans-critical $\mathrm{CO}_{2}$ refrigeration and heat pump cycles in China, he has published over 100 refereed papers with him as the first or corresponding author, including Energy, Energy Conversion and Management Applied Thermal Engineering, International Journal of Heat and Mass Transfer, ASME Journal of Heat Transfer, Progress in Computational Fluid Dynamics, and the relevant research papers in these international academic journals have been widely cited and reviewed by international peers.

\section{SUN Zhi-qiang}

Prof. SUN is with School of Energy Science and Engineering, Central South University, China, and currently he is the dean of the school. Prof. SUN obtained his B.S. and M.S. from Central South University in 2002 and 2004, respectively, and he earned his PhD from Zhejiang University in 2007. During 2011-2012 he worked as a visiting scholar at Duke University, USA. His major research areas include theory and measurement techniques for multiphase flow, thermal design and heat transfer optimization, and new energy technologies. He is the author or co-author of four books and has published more than 100 peer-reviewed papers. Prof. SUN is granted the New Century 121 Talents and Young Talents of Hunan Province, China.

\section{ZHOU Chenn}

Prof. ZHOU obtained her B.S. and M.S. from Nanjing University of Aeronautics and Astronautics in 1982 and 1984, respectively, and $\mathrm{PhD}$ from Carnegie Mellon University in 1991. She jointed Purdue University Northwest in 1994 and became full professor in 2000. Since 2008, she has been a Professor by Courtesy in the School of Mechanical Engineering, Purdue University West Lafayette,
USA. Her current research interests are focusing on innovation through visualization and simulation. In recent years, she has published 9 copyrighted software, 2 patents and more than 350 papers on technical journals, magazines, and conference proceedings. She had been an ASME fellow since 2003.

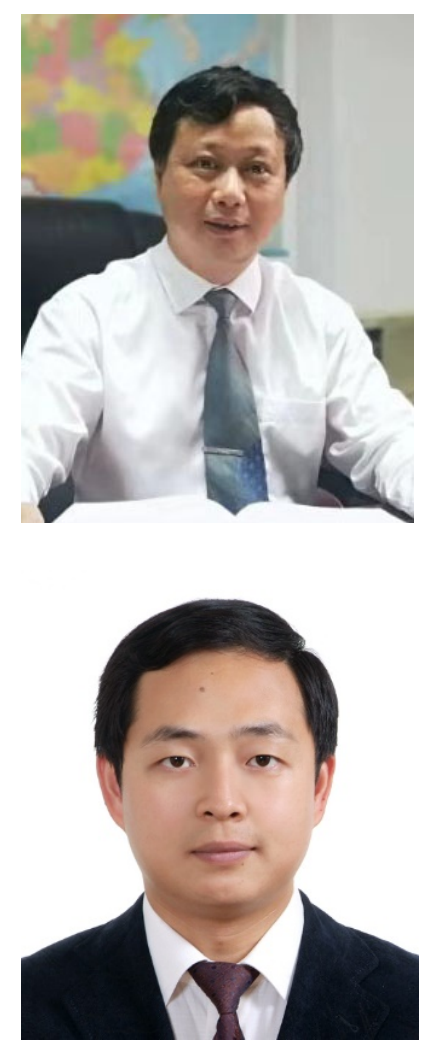

\section{LIAO Sheng-ming}

(PhD, Professor; Tel: +86-731-88836936; E-mail: smliao@csu.edu.cn)

\section{SUN Zhi-qiang}

(PhD, Professor; Tel: +86-731-88879863; E-mail: zqsun@csu. edu.cn; Web of Science Researcher ID: S-3393-2018)

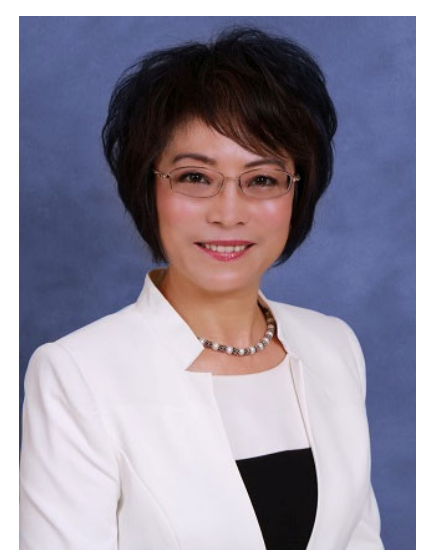

\section{ZHOU Chenn}

(PhD, Professor; Tel: +1-219-989-2665; E-mail: czhou@pnw. edu) 